\title{
Article \\ Facile Fabrication of Magnetic Poly(Vinyl Alcohol)/Activated Carbon Composite Gel for Adsorptive Removal of Dyes
}

\author{
Tao Wen, Baotao Huang and Li Zhou * $\mathbb{D}$
}

check for updates

Citation: Wen, T.; Huang, B.; Zhou, L. Facile Fabrication of Magnetic Poly(Vinyl Alcohol)/Activated Carbon Composite Gel for Adsorptive Removal of Dyes. $J$ Compos. Sci. 2022, 6, 55 https://doi.org/10.3390/jcs6020055

Academic Editor: Ignazio Blanco

Received: 7 January 2022

Accepted: 7 February 2022

Published: 11 February 2022

Publisher's Note: MDPI stays neutral with regard to jurisdictional claims in published maps and institutional affiliations.

Copyright: (C) 2022 by the authors. Licensee MDPI, Basel, Switzerland. This article is an open access article distributed under the terms and conditions of the Creative Commons Attribution (CC BY) license (https:// creativecommons.org/licenses/by/ $4.0 /)$.
Key Laboratory of New Processing Technology for Nonferrous Metal and Materials (Ministry of Education), College of Materials Science and Engineering, Guilin University of Technology, Guilin 541004, China; intervention159@outlook.com (T.W.); huangbaotao1@163.com (B.H.)

* Correspondence: zhouli@glut.edu.cn

\begin{abstract}
Activated carbon (AC) has been widely utilized for the adsorption of pollutants from water. However, it is difficult to recycle the AC after adsorption. In this paper, we report a facile one-pot approach to fabricate magnetic poly(vinyl alcohol)/AC composite gel (mPVA/AC CG) by dropwise addition of an aqueous mixture of PVA, AC and iron ions into the ammonia solution. The obtained mPVA/AC CG after freeze-drying shows porous microstructure and favorable magnetic properties. The utilization of mPVA/AC CG for adsorptive removal of methylene blue (MB) and methyl orange (MO) dyes from water was investigated. The mPVA/AC CG not only exhibited good adsorption performance for both $\mathrm{MB}$ and $\mathrm{MO}$ dyes but also could be readily recycled using a magnet after adsorption. The adsorption process was well described by the pseudo-second-order kinetic model and the Langmuir isotherm model. Considering the simple fabrication process, good adsorption performance and favorable magnetic separation capability, this work provides a viable strategy for combining the features of AC and magnetic gel for fabrication of applicable magnetic adsorbent.
\end{abstract}

Keywords: magnetic gel; activated carbon; adsorption; dye; methylene blue; methyl orange

\section{Introduction}

Dyes are widely used in the textile, paper, rubber, leather, cosmetics, pharmaceutical, food and other industries [1,2]. The widespread use of dyes has led to the production of a large amount of dye wastewater [3,4]. Since dyes are usually difficult to degrade naturally and have mutagenicity and carcinogenicity to aquatic organisms and human body, it is essential to remove dye pollutants from wastewater $[5,6]$. Up to now, adsorption technology has been recognized as a promising dye removal technology because of its high efficiency, convenient operation and wide adaptability to a variety of dyes [7-9]. Activated carbon (AC) has been the most commonly used adsorbent in the past few decades because of its porous structure and large specific surface area [10,11]. However, AC is difficult to recover and reuse after adsorption, which not only causes waste, but also may cause secondary pollution $[12,13]$. Therefore, it is important to develop a feasible method to overcome this limitation of AC adsorbent.

In recent years, magnetic adsorbents have emerged as a new generation of adsorbents for water purification because they can be easily separated from water by an external magnetic field [14,15]. In this regard, the decoration of AC with magnetic nanoparticles to form magnetic AC adsorbent has been widely reported [16-18]. Although the magnetic AC shows good magnetic separation capability and favorable adsorption performance towards various water pollutants (e.g., dyes and metal ions) $[19,20]$, the preparation process is generally tedious and makes it difficult to achieve large-scale production. On the other hand, magnetic nanoparticles easily fall off the surface of AC [21], resulting in a decrease of the magnetic properties of AC. Hence, exploring an effective method to combine the features of $\mathrm{AC}$ and magnetic materials is desired. 
In this work, we report a simple one-pot strategy for fabrication of magnetic poly(vinyl alcohol)/ AC composite gel (mPVA/AC CG) through dropwise addition of an aqueous mixture of PVA, AC and iron ions into ammonia solution (Figure 1). First of all, under the stabilization of polyvinyl alcohol (PVA), iron ions can react with ammonia solution to form magnetic $\mathrm{Fe}_{3} \mathrm{O}_{4}$ nanoparticles. At the same time, the $\mathrm{Fe}_{3} \mathrm{O}_{4}$ nanoparticles can act as a physical cross-linking agent to gelate PVA macromolecules [22]. During the crosslinking process, the $\mathrm{AC}$ was incorporated into the magnetic gel. After freeze-drying, the employment of mPVA/AC CG as a magnetic adsorbent for adsorptive removal of both cationic methylene blue (MB) and anionic methyl orange (MO) dyes was studied. The influence of $\mathrm{AC}$ content, solution $\mathrm{pH}$, contact time and initial dye concentration on the adsorption was investigated systematically.
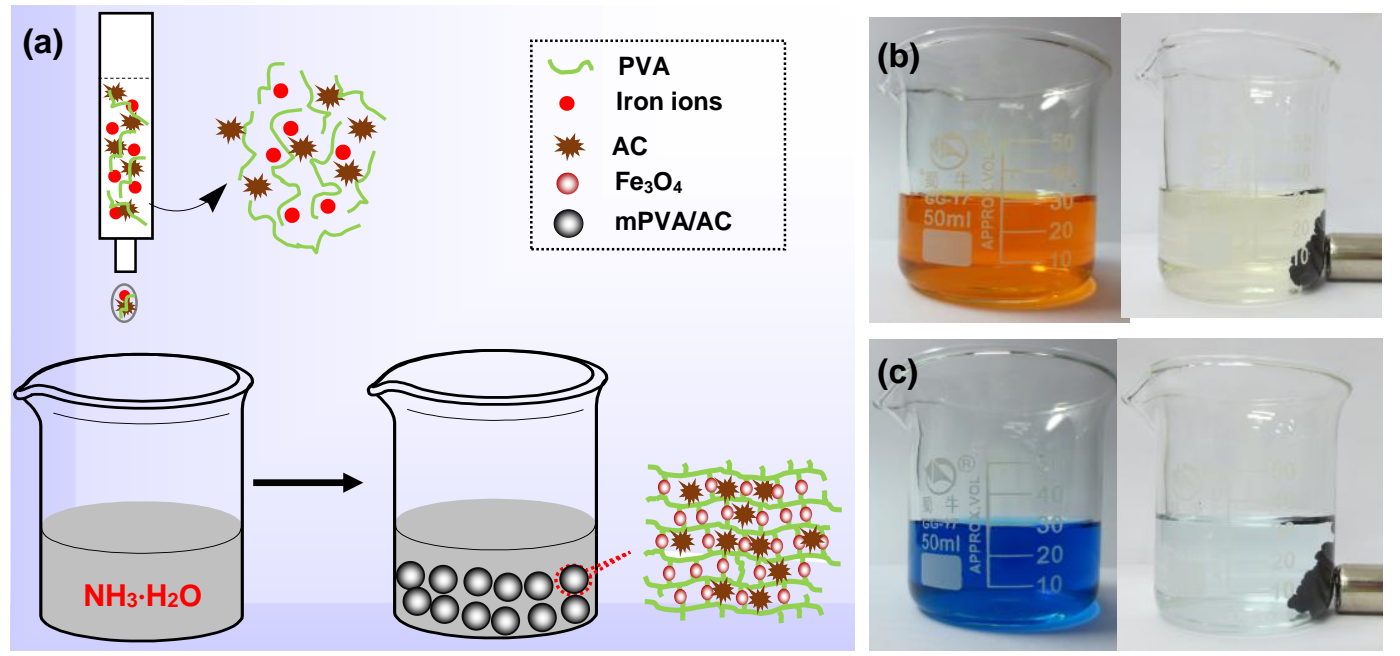

Figure 1. (a) Schematic illustration of the one-pot fabrication of magnetic poly(vinyl alcohol)/activated carbon composite gel (mPVA/AC CG). Photographs of aqueous solution of (b) MO and (c) MB before (left) and after (right) adsorbing by mPVA/AC CG.

\section{Materials and Methods}

\subsection{Materials}

Poly(vinyl alcohol) (PVA, $\mathrm{Mw}=89,000 \mathrm{Da}), \mathrm{FeCl}_{3}$ powder $(99 \%), \mathrm{FeSO}_{4} \cdot 7 \mathrm{H}_{2} \mathrm{O}(99 \%)$, activated carbon (AC, 200 mesh), ammonia solution (25 wt\%), methylene blue (MB, 98\%) and methyl orange (MO) were purchased from Aladdin Chemistry Co. Ltd. (Shanghai, China) and used as received. Deionized water was employed throughout the experiments.

\subsection{Characterization}

Powder X-ray diffraction (XRD) spectra were taken on a Holland PANalytical X-Pert PRO X-ray diffractometer with $\mathrm{Cu}-\mathrm{K} \alpha$ radiation. The magnetization curve was determined on a MPMS XL-7 vibrating-sample magnetometer (VSM) at $300 \mathrm{~K}$. UV-vis absorption spectra were collected on a UV-3600 UV-vis spectrophotometer (Shimadzu). Scanning electron microscopy (SEM) image associated with energy-dispersive X-ray (EDX) spectrum were recorded using a FEI SIRION 200 field emission microscope. Fourier transform infrared (FTIR) spectra were collected on a Thermo Nexus 470 FT-IR spectrometer using a KBr disk.

\subsection{Preparation of $m P V A / A C C G$}

The mPVA/AC CG can be easily fabricated using a one-pot reaction based on the simultaneous formation of $\mathrm{Fe}_{3} \mathrm{O}_{4}$ particles and physical crosslinking of PVA (see Figure 1). To ensure that the mPVA/AC CG possesses a sufficient magnetic separation capability, the theoretical content of $\mathrm{Fe}_{3} \mathrm{O}_{4}$ particles was kept at $20 \mathrm{wt} \%$. The $\mathrm{Fe}_{3} \mathrm{O}_{4}$ particles were formed by co-precipitation of $\mathrm{Fe}^{3+}$ and $\mathrm{Fe}^{2+}$ in ammonia solution and the molar ratio of 
$\mathrm{Fe}^{3+} / \mathrm{Fe}^{2+}$ was kept as constant at 2/1. For mPVA/AC CG with $50 \mathrm{wt} \%$ of AC, $0.695 \mathrm{~g}$ of PVA was dissolved in $20 \mathrm{~mL}$ of deionized water to form a PVA solution. Then, $1.08 \mathrm{~g}$ of $\mathrm{FeCl}_{3}(4.0 \mathrm{mmol})$ and $0.56 \mathrm{~g} \mathrm{FeSO}_{4} \cdot 7 \mathrm{H}_{2} \mathrm{O}(2.0 \mathrm{mmol})$ were added into the PVA solution with stirring at room temperature. Subsequently, $1.16 \mathrm{~g}$ of $\mathrm{AC}$ was added and the mixture was stirred at room temperature for $15 \mathrm{~min}$. Then, the mixture was gradually dropped into $50 \mathrm{~mL}$ of ammonia solution at room temperature to afford black mPVA/AC CG beads. After reaction, the mPVA/AC CG beads were washed with deionized water repeatedly and freeze-dried. For comparison, mPVA/AC CG samples with $0 \mathrm{wt} \%, 30 \mathrm{wt} \%$, and $40 \mathrm{wt} \%$ of $\mathrm{AC}$ were also fabricated in the same condition as above except for the amounts of PVA and AC added.

\subsection{Adsorption Experiments}

The dye adsorption performance of mPVA/AC CG was systematically evaluated by a batch adsorption manner. In this study, $\mathrm{MB}$ and $\mathrm{MO}$ were selected as representative dyes for adsorption experiments (Table 1). The influence of solution $\mathrm{pH}$ was investigated over $\mathrm{pH}$ range of 4-9. The effects of initial dye concentration $(0.1-8.0 \mathrm{mM})$ and contact time were studied at $\mathrm{pH} 7$ and room temperature. In a typical procedure, $20.0 \mathrm{mg}$ of mPVA/AC CG and $5.0 \mathrm{~mL}$ of dye solution with known concentration $(0.1-8.0 \mathrm{mM})$ were mixed by shaking at $100 \mathrm{rpm}$ for specific time in a dark chamber. The adsorption process was monitored using a UV-vis spectrophotometer at room temperature until adsorption equilibrium was reached (about $300 \mathrm{~min}$ ). All adsorption experiments were performed at least three times to minimize random error. Calibration curves were plotted between the concentration of dye solution and absorbance. The adsorption capacity of mPVA/AC CG at equilibrium $\left(Q_{e}\right)(\mathrm{mg} / \mathrm{g})$ was calculated according to the following equation:

$$
\mathrm{Q}_{\mathrm{e}}=\frac{\left(\mathrm{C}_{0}-\mathrm{C}_{\mathrm{e}}\right) \mathrm{V}}{\mathrm{m}}
$$

where $C_{0}$ and $C_{e}$ are the initial and equilibrium concentration of dye $(\mathrm{mg} / \mathrm{L})$, respectively, $\mathrm{V}$ is the volume of the dye solution $(\mathrm{L})$, and $\mathrm{m}(\mathrm{g})$ is the mass of mPVA/AC CG.

Table 1. Structure and characteristics of MB and MO dyes.

Dye

\subsection{Reusability Evaluation}

Regeneration experiments were carried out by immersing the MB-adsorbed mPVA/AC CG into ethanol. The suspension was sonicated in an ultrasonic bath $(100 \mathrm{~W})$ for $30 \mathrm{~min}$. Subsequently, the mPVA/AC CG was separated by a magnet and washed with deionized water. The mPVA/AC CG was freeze-dried again for adsorption.

\section{Results and Discussions}

\subsection{Preparation and Characterization of $m P V A / A C C G$}

The one-pot reaction for fabrication of mPVA/AC CG is based on the simultaneous formation of magnetic $\mathrm{Fe}_{3} \mathrm{O}_{4}$ nanoparticles, cross-linking of PVA macromolecules and incorporation of AC (Figure 1). Upon dropwise addition of the mixture of PVA, AC and iron ions into the ammonia solution, black mPVA/AC CG beads were formed immediately. The size of mPVA/AC CG beads was in the range of 1.5-3.0 mm (Figure 2a). The morphology of freeze-dried mPVA/AC CG was observed by SEM. As can be seen in Figure $2 b, c$, the surface 
morphology of mPVA/AC CG was similar to that of AC, indicating that the microstructure of $\mathrm{AC}$ was well retained during the reaction process. An obvious porous microstructure can be seen in the mPVA/AC CG sample. It is expected that these pores promote the transport of dye molecules during the adsorption process. In addition, an obvious Fe element signal can be observed in the EDX spectrum of the mPVA/AC CG (Figure 2d), which is consistent with the expected composition of mPVA/AC CG.
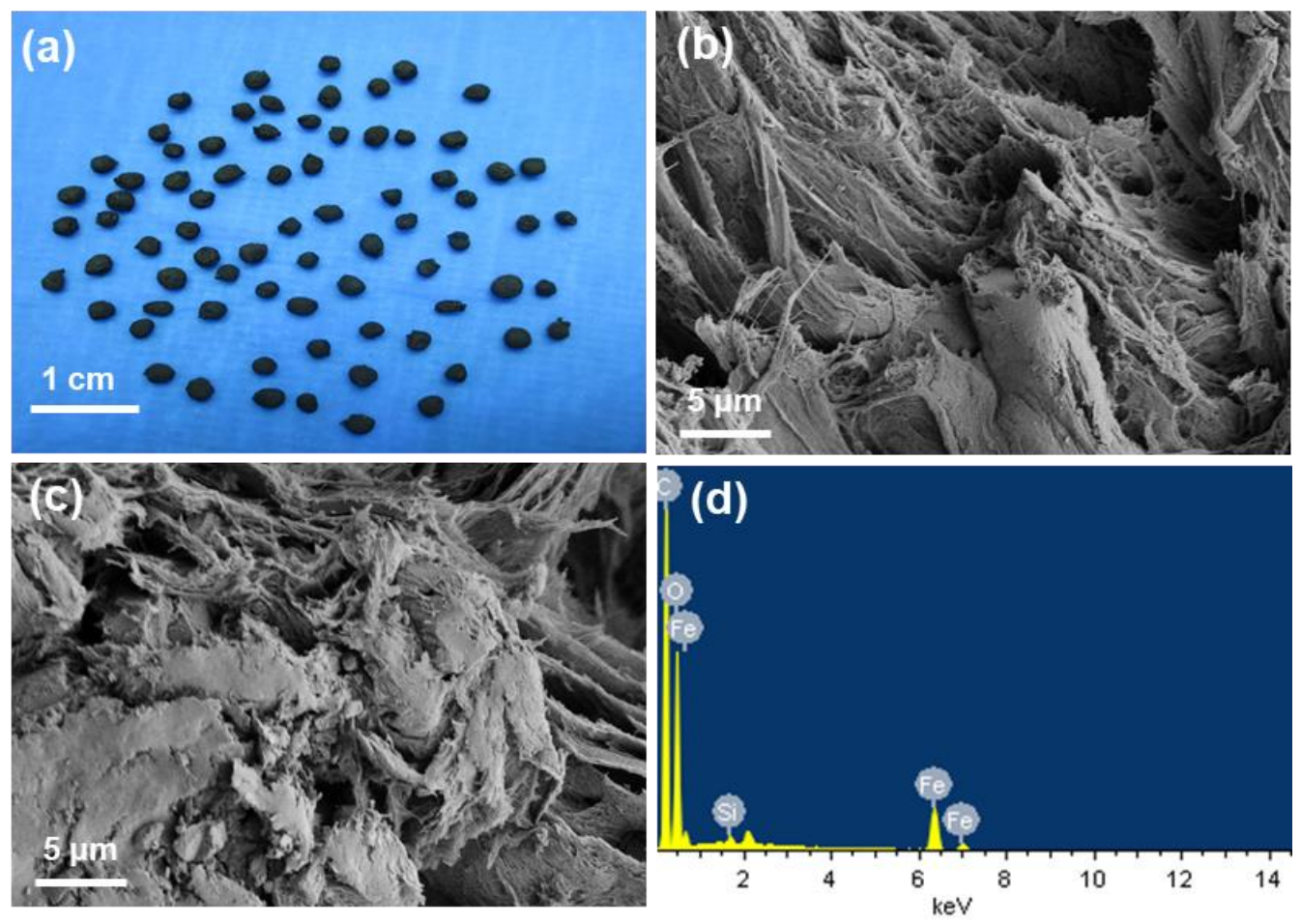

Figure 2. (a) Photograph of mPVA/AC CG beads. (b) SEM image of AC. (c) SEM image and (d) EDX spectrum of mPVA/AC CG sample.

To further investigate the structure of mPVA/AC CG, a powder XRD measurement was performed. As shown in Figure $3 a$, the wide diffraction peak at $2 \theta=19.7^{\circ}$ is the characteristic peak of semicrystalline PVA. The diffraction patterns and relative intensities of other peaks matched well with that of $\mathrm{Fe}_{3} \mathrm{O}_{4}$ (JCPDS 19-0629), indicating that $\mathrm{Fe}_{3} \mathrm{O}_{4}$ particles were formed in the mPVA/AC CG [23]. Additionally, the magnetic property of mPVA/AC CG was determined by VSM at $300 \mathrm{~K}$. The mPVA/AC CG exhibited a favorable superparamagnetic behavior with a saturation magnetization value of about $7.3 \mathrm{emu} / \mathrm{g}$ (Figure $3 b$ ), which promised a good magnetic separation capability as confirmed in the inset of Figure $3 b$.

\subsection{Adsorption Performance of $m P V A / A C C G$}

The adsorption performance of bead-like mPVA/AC CG was studied using MB and $\mathrm{MO}$ as two typical dyes. The chemical structures of MB and MO are presented in Table 1. As shown in Figure $4 a, b$, the aqueous solutions of $\mathrm{MB}$ and $\mathrm{MO}$ have obvious absorption peaks at $662 \mathrm{~nm}$ and $463 \mathrm{~nm}$, respectively. After mixing mPVA/AC CG, the intensity of these peaks decreased gradually, indicating that mPVA/AC CG adsorbent can effectively adsorb MB and MO molecules from water. To further probe the adsorption performance of mPVA/AC CG, the effects of $\mathrm{AC}$ content, solution $\mathrm{pH}$, contact time, and initial dye concentration were studied systematically. 

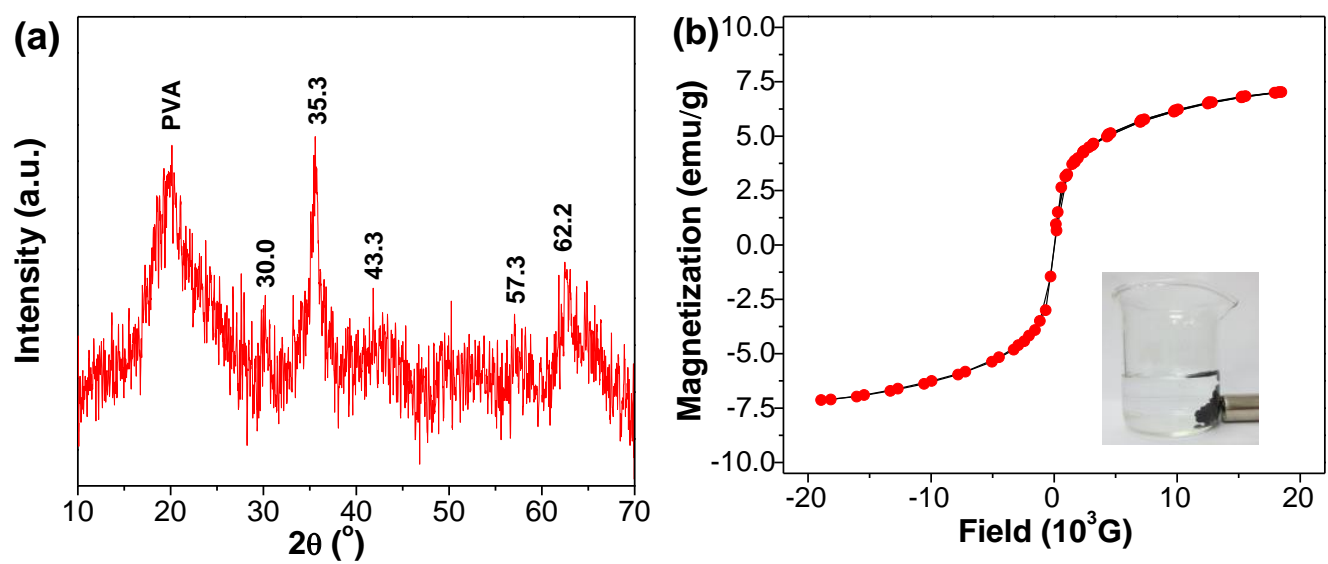

Figure 3. (a) Powder XRD pattern of mPVA/AC CG. (b) Magnetization curve ( $300 \mathrm{~K})$ of mPVA/AC CG. Inset: photograph of mPVA/AC CG beads in water after placement of a magnet.
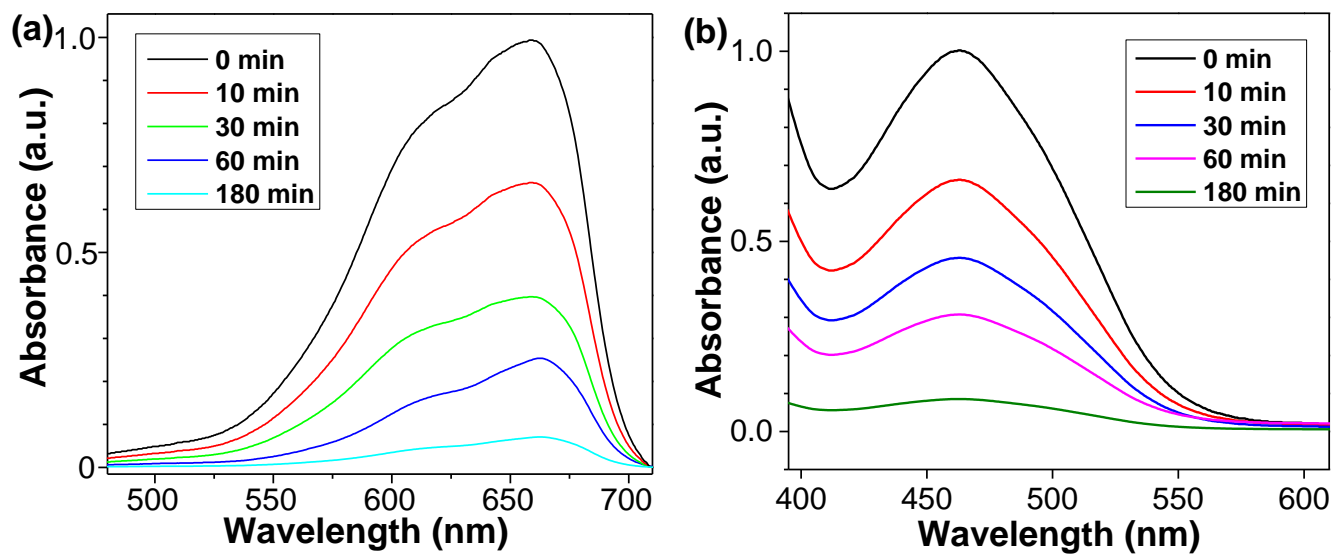

Figure 4. UV-vis absorption spectra of (a) MB and (b) MO after mixing mPVA/AC CG with 50 wt $\%$ of $\mathrm{AC}$ at $\mathrm{pH} 7$ for different times. The initial dye concentration was $0.1 \mathrm{mM}$.

First, the influence of AC content on the adsorption capacity of mPVA/AC CG was investigated. For comparison, the adsorption capacity of AC was also determined with the same condition. At a relatively high initial dye concentration $(5.0 \mathrm{mM})$, the saturated adsorption capacities of mPVA/AC CG for both MB and MO increased obviously, with an increase in the content of AC (Figure 5a). For example, the adsorption capacities of mPVA/AC CG samples with $0 \mathrm{wt} \%, 30 \mathrm{wt} \%, 40 \mathrm{wt} \%$ and $50 \mathrm{wt} \%$ of AC towards MB were $94.3 \mathrm{mg} / \mathrm{g}, 137.6 \mathrm{mg} / \mathrm{g}, 150.2 \mathrm{mg} / \mathrm{g}$ and $172.1 \mathrm{mg} / \mathrm{g}$, respectively. It is worth noting that it is difficult to obtain structurally stable mPVA/AC CG by further increasing the content of AC, because too little PVA cannot form a stable magnetic gel. Therefore, the mPVA/AC CG sample with $50 \mathrm{wt} \%$ of $\mathrm{AC}$ was chosen for the subsequent adsorption experiments. Although the adsorption capacity of the mPVA/AC CG sample was lower than that of AC, it could be easily collected by a magnet after adsorption. This feature is highly desirable for practical adsorption applications.

Since the interactions between dye molecules and adsorbent are heavily affected by solution $\mathrm{pH}[24,25]$, the effect of $\mathrm{pH}$ on the adsorption performance of mPVA/AC CG was assessed in the $\mathrm{pH}$ range of 4-9. As depicted in Figure $5 \mathrm{~b}$, the variation of solution $\mathrm{pH}$ has slight influence on the adsorption capacity of mPVA/AC CG towards both MB and MO. This result suggests that changing the surface charges of dye molecules and adsorbent has negligible effect on the adsorption. Therefore, the main driving force behind dye adsorption by mPVA/AC CG is not electrostatic interaction, but by molecular interactions (e.g., hydrogen bonding and van der Waals force) [26,27]. 

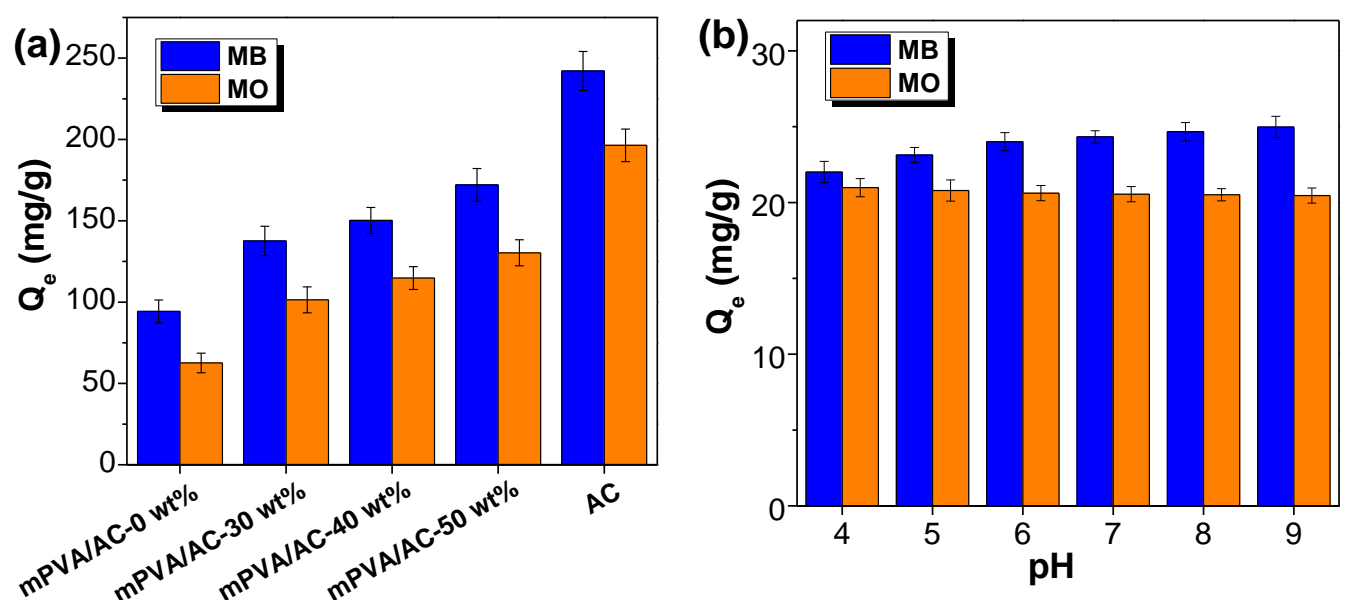

Figure 5. (a) Adsorption capacities of AC and mPVA/AC CG samples with different content of AC for adsorption of $\mathrm{MB}$ and $\mathrm{MO}$. The initial dye concentration was $5 \mathrm{mM}$. (b) Effect of solution $\mathrm{pH}$ on the adsorption of MB and MO by mPVA/AC CG. The initial dye concentration was $0.1 \mathrm{mM}$.

The effect of contact time on the uptake of MB and MO by mPVA/AC CG was studied with $20 \mathrm{mg}$ of mPVA/AC CG in $5 \mathrm{~mL}$ of $0.1 \mathrm{mM}$ dye at $\mathrm{pH} 7$. As presented in Figure 6a, the mPVA/AC CG exhibited a fast adsorption rate during the initial $30 \mathrm{~min}$ for both MB and MO dyes. The whole adsorption process reached equilibrium within about $300 \mathrm{~min}$. The rapid adsorption in the initial stage was due to the interactions between dye molecules and binding sites on the outer surface of mPVA/AC CG. When these binding sites reach saturation, the binding sites on the inner surface of mPVA/AC CG begin to participate in the adsorption of dye molecules, resulting in a relatively slow increase in adsorption capacity [28]. To further probe the adsorption mechanism, the adsorption data were analyzed using two important kinetic models including pseudo-first-order and pseudosecond-order kinetic models [29,30]. The values of kinetic parameters were calculated based on the slope and intercept of the corresponding fitting curve (Figure $6 b, c$ ) and the results are illustrated in Table 2. As can be seen in Figure $6 \mathrm{~b}, \mathrm{c}$, the pseudo-second-order kinetic model possesses much higher correlation coefficients $\left(R^{2}\right)$ values $(0.999$ and 0.994 for $\mathrm{MB}$ and $\mathrm{MO}$, respectively) than that of the pseudo-first-order kinetic model $(0.972$ and 0.990 for $\mathrm{MB}$ and $\mathrm{MO}$, respectively). Furthermore, the calculated $Q_{\mathrm{e}}$ values (25.80 and $22.05 \mathrm{mg} / \mathrm{g}$ for MB and MO, respectively) based on the pseudo-second-order kinetic model are similar to the experimental $Q_{\mathrm{e}}$ values $(24.34$ and $20.55 \mathrm{mg} / \mathrm{g}$ for $\mathrm{MB}$ and MO, respectively) (Table 2). All these results suggest that the adsorption process obeyed the pseudo-second-order kinetic model, which means that chemisorption occurred.
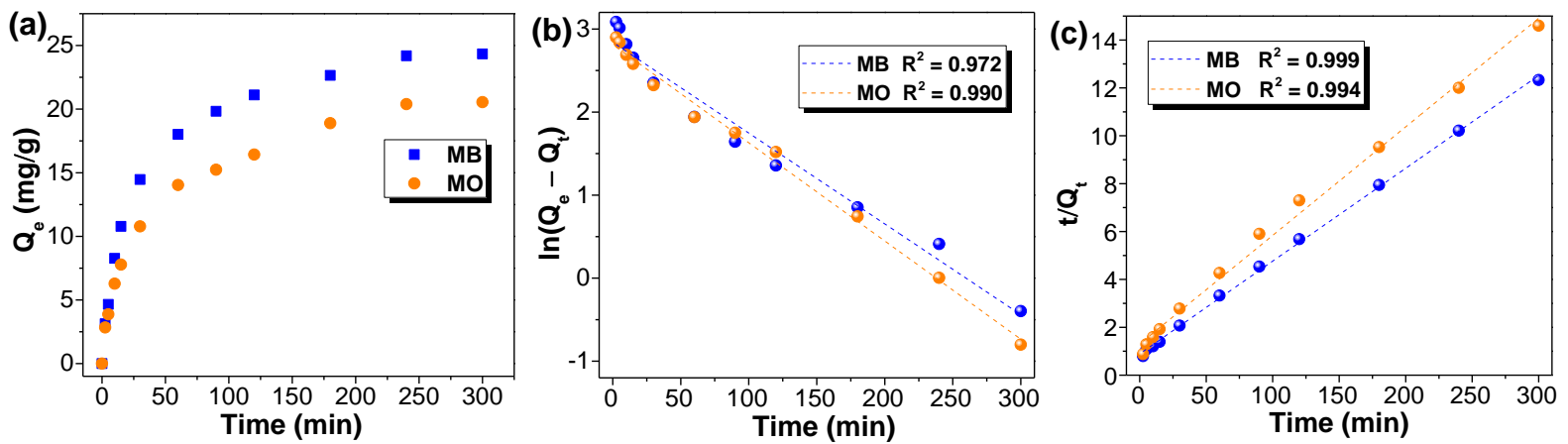

Figure 6. (a) Effect of contact time on the adsorption of MB and MO by mPVA/AC CG at $\mathrm{pH}$ 7. The initial dye concentration was $0.1 \mathrm{mM}$. (b) Plots of $\ln \left(\mathrm{Q}_{\mathrm{e}}-\mathrm{Q}_{\mathrm{t}}\right)$ against $\mathrm{t}$ based on the pseudo-first-order kinetic model. (c) Plots of $t / Q_{t}$ against $t$ based on the pseudo-second-order kinetic model. 
Table 2. The kinetic parameters for the adsorption of MB and MO onto the mPVA/AC CG.

\begin{tabular}{|c|c|c|c|c|c|c|c|}
\hline & \multirow[b]{2}{*}{$\begin{array}{l}\text { Qe-exp } \\
(\mathrm{mg} / \mathrm{g})\end{array}$} & \multicolumn{3}{|c|}{$\begin{array}{c}\text { Pseudo-First-Order } \\
\ln \left(Q_{e}-Q_{t}\right)=\ln Q_{e}-k_{1} t\end{array}$} & \multicolumn{3}{|c|}{$\begin{array}{l}\text { Pseudo-Second-Order } \\
t / Q_{t}=1 / k_{2} Q^{2}{ }_{e}+t / Q_{e}\end{array}$} \\
\hline & & $\begin{array}{c}\mathrm{Q}_{\mathrm{e}-\mathrm{cal}} \\
(\mathrm{mg} / \mathrm{g})\end{array}$ & $\begin{array}{c}\mathrm{k}_{1} \\
\left(\min ^{-1}\right)\end{array}$ & $\mathrm{R}^{2}$ & $\begin{array}{c}\mathrm{Q}_{\mathrm{e}-\mathrm{cal}} \\
(\mathrm{mg} / \mathrm{g})\end{array}$ & $\left(\mathrm{mg}^{-1} \mathrm{~min}^{-1}\right)$ & $\mathrm{R}^{2}$ \\
\hline $\mathrm{MB}$ & $24.34 \pm 0.62$ & $29.02 \pm 0.15$ & $0.028 \pm 0.003$ & 0.972 & $25.80 \pm 0.94$ & $0.0017 \pm 0.0001$ & 0.999 \\
\hline $\mathrm{MO}$ & $20.55 \pm 0.39$ & $16.65 \pm 0.13$ & $0.012 \pm 0.002$ & 0.990 & $22.05 \pm 0.75$ & $0.0016 \pm 0.0001$ & 0.994 \\
\hline
\end{tabular}

In order to further understand the interaction between mPVA/AC CG and dyes, the equilibrium adsorption isotherm of mPVA/AC CG was studied. As depicted in Figure 7a, the $Q_{e}$ values of mPVA/AC CG for both MB and MO dyes were enhanced when increasing the equilibrium concentration associated with the initial dye concentration. This is because a higher initial concentration can not only provide sufficient driving force to overcome the mass transfer resistance of dye molecules from aqueous phase to mPVA/AC CG phase, but also provide more opportunities for dye molecules to come into contact with the binding sites of mPVA/AC CG. Two well-known models, Langmuir and Freundlich isotherm models were employed to analyze the isotherm data [31,32]. The Langmuir isotherm assumes that the surface of the adsorbent has homogeneous binding sites with identical adsorption energies, while the Freundlich isotherm model assumes that the surface of the adsorbent is heterogeneous in nature. The fitting results and the calculated isotherm constants are shown in Figure 7b,c and Table 3. Clearly, the Langmuir isotherm model exhibits higher $\mathrm{R}^{2}$ value of 0.992 and 0.990 for uptake of MB and MO, respectively, and, thus, it is more suitable for description of the adsorption process compared with the Freundlich isotherm model. Therefore, the adsorption of MB and MO molecules onto the mPVA/AC CG occurred at specific homogeneous binding sites. A monolayer coverage of $\mathrm{MB}$ or $\mathrm{MO}$ was formed on the surface of mPVA/AC CG. The maximum adsorption capacity $\left(\mathrm{Q}_{\mathrm{m}}\right)$ of mPVA/AC CG for MB and MO based on the Langmuir isotherm model was calculated to be 173.91 and $146.63 \mathrm{mg} / \mathrm{g}$, respectively, which is higher than many other reported magnetic adsorbents (see Table 4) [33-40] such as magnetic graphene oxide (64.23 mg/g for MB) [33], magnetic carbon nanotubes (15.74 mg/g for MB) [34], magnetic rectorite $/ \mathrm{Fe}_{3} \mathrm{O}_{4}$ composites (60.24 mg/g for MB) [35], magnetic $\gamma$ $\mathrm{Fe}_{2} \mathrm{O}_{3} @ G L$ (69.93-91.74 mg/g for MB) [36], magnetic lignin-based carbon nanoparticles (113.00 mg/g for MO) [38], and magnetic $\mathrm{LDH} @ \mathrm{Fe}_{3} \mathrm{O}_{4} / \mathrm{PVA}$ composites (19.59 mg/g for MO) [39].
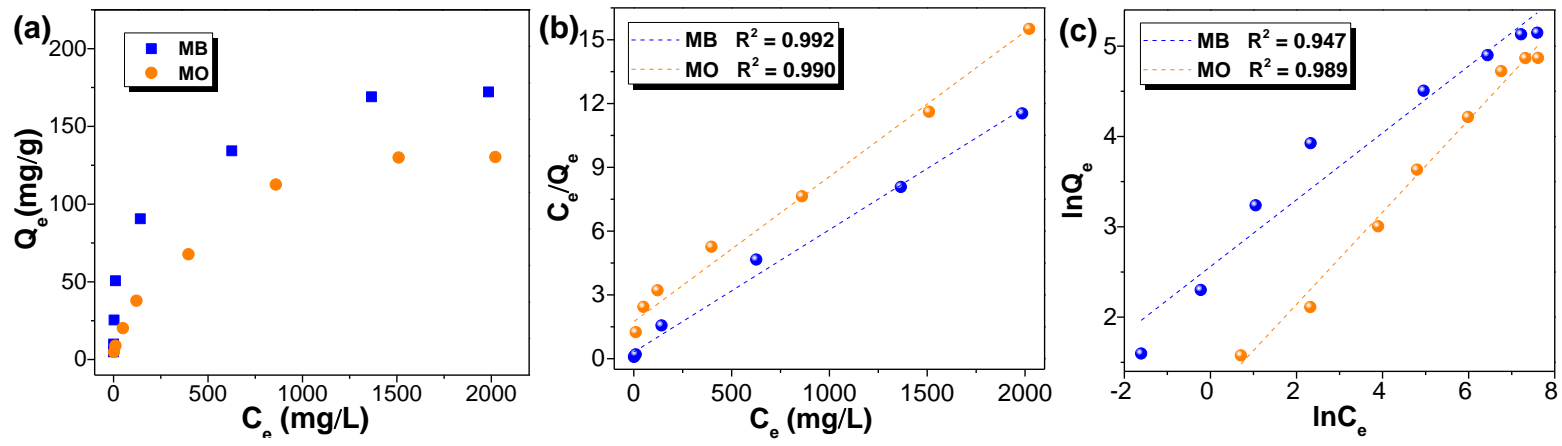

Figure 7. (a) Adsorption isotherms for the adsorption of MB and MO by mPVA/AC CG at $\mathrm{pH} 7$. (b) The values of $C_{e} / Q_{e}$ against $C_{e}$ based on the Langmuir isotherm model. (c) The values of $\ln Q_{e}$ against $\ln C_{\mathrm{e}}$ based on the Freundlich isotherm model. 
Table 3. The isotherm parameters for the adsorption of MB and MO onto the mPVA/AC CG.

\begin{tabular}{cccc}
\hline Isotherm Model & Parameter & MB & MO \\
\hline Langmuir: $\mathrm{C}_{\mathrm{e}} / \mathrm{Q}_{\mathrm{e}}=\mathrm{C}_{\mathrm{e}} / \mathrm{Q}_{\mathrm{m}}+1 / \mathrm{K}_{\mathrm{L}} \mathrm{Q}_{\mathrm{m}}$ & $\mathrm{Q}_{\mathrm{m}}(\mathrm{mg} / \mathrm{g})$ & $173.91 \pm 1.6$ & $146.63 \pm 1.3$ \\
& $\mathrm{~K}_{\mathrm{L}}(\mathrm{L} / \mathrm{mg})$ & $0.018 \pm 0.002$ & $0.004 \pm 0.001$ \\
& $\mathrm{R}^{2}$ & 0.992 & 0.990 \\
\cline { 2 - 4 } Freundlich: $\ln \mathrm{Q}_{\mathrm{e}}=\ln \mathrm{K}_{\mathrm{F}}+\mathrm{b}_{\mathrm{F}} \ln \mathrm{C}_{\mathrm{e}}$ & $\mathrm{K}_{\mathrm{F}}(\mathrm{mg} / \mathrm{g})$ & $12.93 \pm 0.21$ & $3.065 \pm 0.038$ \\
& $\mathrm{~b}_{\mathrm{F}}$ & $0.370 \pm 0.031$ & $0.511 \pm 0.022$ \\
& $\mathrm{R}^{2}$ & 0.947 & 0.989 \\
\hline
\end{tabular}

Table 4. Comparison of the maximum monolayer adsorption of $\mathrm{MB}$ and $\mathrm{MO}$ onto various magnetic adsorbents.

\begin{tabular}{|c|c|c|c|}
\hline Dye & Adsorbent & $\underset{(\mathrm{mg} / \mathrm{g})}{Q_{\max }}$ & Reference \\
\hline $\mathrm{MB}$ & Magnetic GO & 64.23 & 33 \\
\hline $\mathrm{MB}$ & Magnetic carbon nanotube & 15.74 & 34 \\
\hline MB & Magnetic rectorite $/ \mathrm{Fe}_{3} \mathrm{O}_{4}$ composites & 60.24 & 35 \\
\hline MB & Magentic $\gamma-\mathrm{Fe}_{2} \mathrm{O}_{3} @ G L$ & $69.93-91.74$ & 36 \\
\hline MB & Magnetic $\mathrm{GO}-\mathrm{Fe}_{3} \mathrm{O}_{4}$ hybrid & 167.3 & 37 \\
\hline $\mathrm{MB}$ & mPVA/AC CG & 173.91 & This study \\
\hline $\mathrm{MO}$ & $\begin{array}{l}\text { Magnetic lignin-based carbon } \\
\text { nanoparticles }\end{array}$ & 113.00 & 38 \\
\hline $\mathrm{MO}$ & Magnetic LDH@ $\mathrm{Fe}_{3} \mathrm{O}_{4} /$ PVA composites & 19.59 & 39 \\
\hline $\mathrm{MO}$ & Chitosan modified magnetic kaolin & 349.70 & 40 \\
\hline $\mathrm{MO}$ & $\mathrm{mPVA} / \mathrm{AC} C \mathrm{G}$ & 146.63 & This study \\
\hline
\end{tabular}

To better understand the adsorption mechanism, FTIR spectra of mPVA/AC CG before and after uptake of MB and MO were recorded. In the FTIR spectrum of MPVA/AC CG (Figure 8), the characteristic peaks at about 3412 and $1109 \mathrm{~cm}^{-1}$ were assigned to the $-\mathrm{OH}$ group and C-O bond of PVA, respectively. The two bands at 2942 and $2861 \mathrm{~cm}^{-1}$ were assigned to the stretching vibration of the $\mathrm{C}-\mathrm{H}$ bond. After adsorption of $\mathrm{MB}$ and $\mathrm{MO}$, the characteristic absorption bands corresponding to the vibrations of the aromatic ring (e.g., 1600 and $823 \mathrm{~cm}^{-1}$ ) and C-N bond (e.g., $1318 \mathrm{~cm}^{-1}$ ) appeared (Figure 8) [41,42], indicating the successful uptake of MB and MO dyes by mPVA/AC CG.

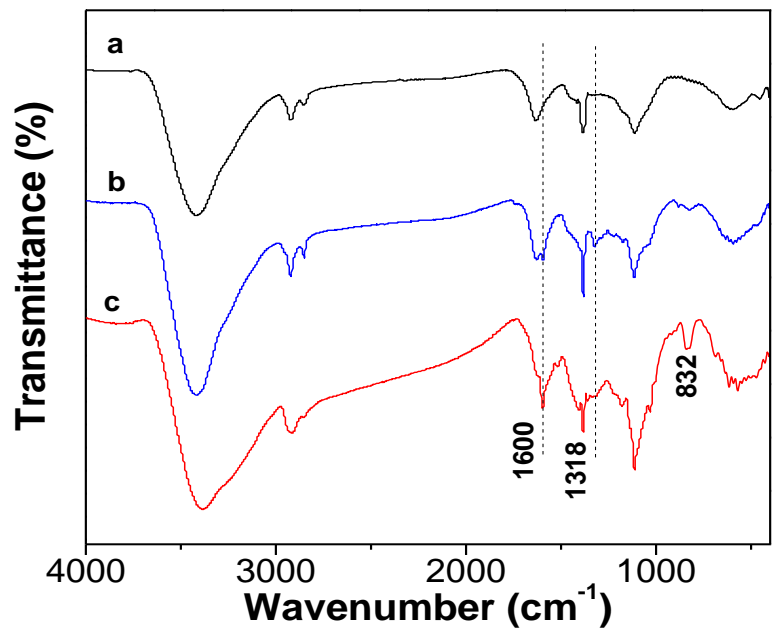

Figure 8. FTIR spectra of mPVA/AC CG (a), MB-adsorbed mPVA/AC CG (b) and MO-adsorbed mPVA/AC CG (c). 
For real adsorption application, an adsorbent with good reusability is highly desirable. In this work, the reusability of MB-adsorbed mPVA/AC CG was assessed by employing ethanol as an eluting agent. As can be seen in Figure 9a, the removal efficiency of desorbed mPVA/AC CG decreased with the increase of reuse times. Compared with the original removal efficiency, the removal efficiency remained about $72.1 \%$ after five cycles of desorption-adsorption. Nevertheless, the adsorption capacity of regenerated mPVA/AC CG could still reach $155.39 \mathrm{mg} / \mathrm{g}$, which is higher than many reported magnetic adsorbents [33-36]. In addition, even after five cycles of regeneration-adsorption, the mPVA/AC CG still exhibited similar a saturation magnetization value to that of the newly prepared one (Figure 9b), indicating the good structural stability of mPVA/AC CG.
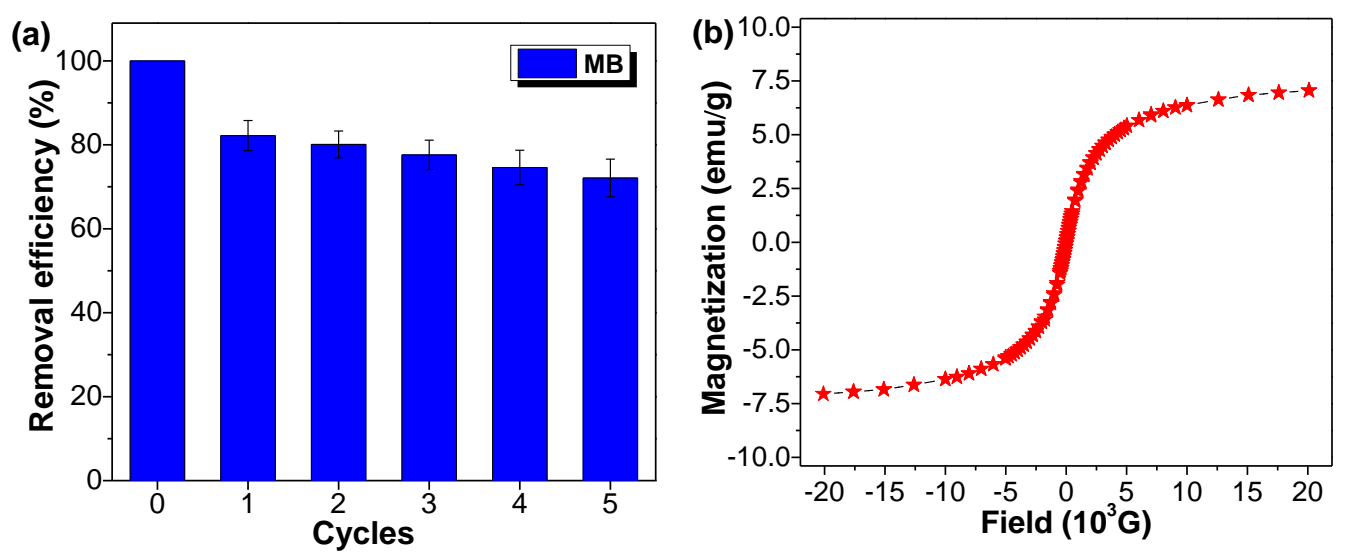

Figure 9. (a) Removal efficiency of mPVA/AC CG adsorbent in five successive cycles of desorptionadsorption. (b) Magnetization curve (300 K) of mPVA/AC CG adsorbent after reuse for five times.

\section{Conclusions}

In summary, we have demonstrated a facile one-pot strategy for fabrication of mPVA/AC CG based on the simultaneous formation of a magnetic PVA gel and incorporation of AC. Due to its porous microstructure and favorable magnetic property, the mPVA/AC CG showed good adsorption performance and favorable magnetic separation capability for adsorptive removal of $\mathrm{MB}$ and $\mathrm{MO}$ dyes from water. The adsorption data fitted well with the pseudo-second-order kinetic and Langmuir isotherm models. Compared with previously reported AC adsorbents and magnetic gel adsorbents, this study highlights the combined merits of $\mathrm{AC}$ and magnetic gel, consequently offering an effective approach for preparing an applicable magnetic adsorbent for the practical removal of dye pollutants from wastewater.

Author Contributions: Conceptualization, T.W. and L.Z.; investigation, T.W. and B.H.; supervision, L.Z.; writing-original draft, T.W. All authors have read and agreed to the published version of the manuscript.

Funding: This work was supported by the Bagui Scholar Program of Guangxi Province.

Data Availability Statement: The data presented in this study are available on request from the corresponding author.

Conflicts of Interest: The authors declare no conflict of interest.

\section{References}

1. Gao, J.J.; Qin, Y.B.; Zhou, T.; Cao, D.D.; Xu, P.; Hochstetter, D.; Wang, Y.F. Adsorption of methylene blue onto activated carbon produced from tea (Camellia sinensis L.) seed shells: Kinetics, equilibrium, and thermodynamics studies. J. Zhejiang Univ. Sci. B 2013, 14, 650-658. [CrossRef] [PubMed]

2. Munir, M.; Nazar, M.F.; Zafar, M.N.; Zubair, M.; Ashfaq, M.; Hosseini-Bandegharaei, A.; Khan, S.U.D.; Ahmad, A. Effective adsorptive removal of methylene blue from water by didodecyldimethylammonium bromide-modified Brown clay. ACS Omega 2020, 5, 16711-16721. [CrossRef] [PubMed] 
3. Li, H.; Liu, L.; Cui, J.; Cui, J.; Wang, F.; Zhang, F. High-efficiency adsorption and regeneration of methylene blue and aniline onto activated carbon from waste edible fungus residue and its possible mechanism. RSC Adv. 2020, 10, 14262-14273. [CrossRef]

4. Das, T.R.; Sharma, P.K. Bimetal oxide decorated graphene oxide $\left(\mathrm{Gd}_{2} \mathrm{O}_{3} / \mathrm{Bi}_{2} \mathrm{O}_{3} @ G O\right)$ nanocomposite as an excellent adsorbent in the removal of methyl orange dye. Mater. Sci. Semicon. Proc. 2020, 105, 104721. [CrossRef]

5. Duhan, M.; Kaur, R. Nano-structured polyaniline as a potential adsorbent for methylene blue dye removal from effluent. J. Compos. Sci. 2021, 5, 7. [CrossRef]

6. El-Shamy, A.G. An efficient removal of methylene blue dye by adsorption onto carbon dot@ zinc peroxide embedded poly vinyl alcohol (PVA $/ \mathrm{CZnO}_{2}$ ) nano-composite: A novel Reusable adsorbent. Polymer 2020, 202, 122565. [CrossRef]

7. Kumar, P.S.; Joshiba, G.J.; Femina, C.C.; Varshini, P.; Priyadharshini, S.; Karthick, M.A.; Jothirani, R. A critical review on recent developments in the low-cost adsorption of dyes from wastewater. Desalin. Water Treat 2019, 172, 395-416. [CrossRef]

8. Başar, C.A. Applicability of the various adsorption models of three dyes adsorption onto activated carbon prepared waste apricot. J. Hazard. Mater. 2006, 135, 232-241. [CrossRef]

9. Yang, J.; Qiu, K. Preparation of activated carbons from walnut shells via vacuum chemical activation and their application for methylene blue removal. Chem. Eng. J. 2010, 165, 209-217. [CrossRef]

10. Naushad, M.; Alqadami, A.A.; AlOthman, Z.A.; Alsohaimi, I.H.; Algamdi, M.S.; Aldawsari, A.M. Adsorption kinetics, isotherm and reusability studies for the removal of cationic dye from aqueous medium using arginine modified activated carbon. J. Mol. Liq. 2019, 293, 111442. [CrossRef]

11. Boudechiche, N.; Fares, M.; Ouyahia, S.; Yazid, H.; Trari, M.; Sadaoui, Z. Comparative study on removal of two basic dyes in aqueous medium by adsorption using activated carbon from Ziziphus lotus stones. Microche. J. 2019, 146, 1010-1018. [CrossRef]

12. Badi, M.Y.; Azari, A.; Pasalari, H.; Esrafili, A.; Farzadkia, M. Modification of activated carbon with magnetic $\mathrm{Fe}_{3} \mathrm{O}_{4}$ nanoparticle composite for removal of ceftriaxone from aquatic solutions. J. Mol. Liq. 2018, 261, 146-154. [CrossRef]

13. Bystrzejewski, M.; Pyrzyńska, K. Kinetics of copper ions sorption onto activated carbon, carbon nanotubes and carbonencapsulated magnetic nanoparticles. Colloid. Surf. A 2011, 377, 402-408. [CrossRef]

14. Lin, Y.F.; Chen, H.W.; Chien, P.S.; Chiou, C.S.; Liu, C.C. Application of bifunctional magnetic adsorbent to adsorb metal cations and anionic dyes in aqueous solution. J. Hazard. Mater. 2011, 185, 1124-1130. [CrossRef] [PubMed]

15. Song, Y.; Duan, Y.; Zhou, L. Multi-carboxylic magnetic gel from hyperbranched polyglycerol formed by thiol-ene photopolymerization for efficient and selective adsorption of methylene blue and methyl violet dyes. J. Colloid Interface Sci. 2018, 529, 139-149. [CrossRef] [PubMed]

16. Ebadollahzadeh, H.; Zabihi, M. Competitive adsorption of methylene blue and $\mathrm{Pb}$ (II) ions on the nano-magnetic activated carbon and alumina. Mater. Chem. Phys. 2020, 248, 122893. [CrossRef]

17. Cazetta, A.L.; Pezoti, O.; Bedin, K.C.; Silva, T.L.; Paesano Junior, A.; Asefa, T.; Almeida, V.C. Magnetic activated carbon derived from biomass waste by concurrent synthesis: Efficient adsorbent for toxic dyes. ACS Sustain. Chem. Eng. 2016, 4, 1058-1068. [CrossRef]

18. Altintig, E.; Onaran, M.; Sarı, A.; Altundag, H.; Tuzen, M. Preparation, characterization and evaluation of bio-based magnetic activated carbon for effective adsorption of malachite green from aqueous solution. Mater. Chem. Phys. 2018, 220, 313-321. [CrossRef]

19. Zhang, Z.; Wang, T.; Zhang, H.; Liu, Y.; Xing, B. Adsorption of Pb (II) and Cd (II) by magnetic activated carbon and its mechanism. Sci. Total Environ. 2021, 757, 143910. [CrossRef]

20. Guo, F.; Li, X.; Jiang, X.; Zhao, X.; Guo, C.; Rao, Z. Characteristics and toxic dye adsorption of magnetic activated carbon prepared from biomass waste by modified one-step synthesis. Colloid. Surf. A 2018, 555, 43-54. [CrossRef]

21. Rocha, L.S.; Pereira, D.; Sousa, É.; Otero, M.; Esteves, V.I.; Calisto, V. Recent advances on the development and application of magnetic activated carbon and char for the removal of pharmaceutical compounds from waters: A review. Sci. Total Environ. 2020, 718, 137272. [CrossRef] [PubMed]

22. Li, C.; Wang, X.; Meng, D.; Zhou, L. Facile synthesis of low-cost magnetic biosorbent from peach gum polysaccharide for selective and efficient removal of cationic dyes. Int. J. Bio. Macromol. 2018, 107, 1871-1878. [CrossRef] [PubMed]

23. Chen, J.; Huang, K.; Liu, S. Hydrothermal preparation of octadecahedron $\mathrm{Fe}_{3} \mathrm{O}_{4}$ thin film for use in an electrochemical supercapacitor. Electrochim. Acta 2009, 55, 1-5. [CrossRef]

24. Kong, D.; Wilson, L.D. Uptake of methylene blue from aqueous solution by pectin-chitosan binary composites. J. Compos. Sci. 2020, 4, 95. [CrossRef]

25. Song, Y.; Tan, J.; Wang, G.; Zhou, L. Superior amine-rich gel adsorbent from peach gum polysaccharide for highly efficient removal of anionic dyes. Carbohydr. Polym. 2018, 199, 178-185. [CrossRef]

26. Crini, G. Studies on adsorption of dyes on beta-cyclodextrin polymer. Bioresour. Technol. 2003, 90, 193-198. [CrossRef]

27. Qiu, Y.; Zheng, Z.; Zhou, Z.; Sheng, G.D. Effectiveness and mechanisms of dye adsorption on a straw-based biochar. Bioresour. Technol. 2009, 100, 5348-5351. [CrossRef]

28. Hu, X.S.; Liang, R.; Sun, G. Super-adsorbent hydrogel for removal of methylene blue dye from aqueous solution. J. Mater. Chem. A 2018, 6, 17612-17624. [CrossRef]

29. Song, Y.B.; Lv, S.N.; Cheng, C.J.; Ni, G.L.; Xie, X.W.; Huang, W.; Zhao, Z.G. Fast and highly-efficient removal of methylene blue from aqueous solution by poly (styrenesulfonic acid-co-maleic acid)-sodium-modified magnetic colloidal nanocrystal clusters. Appl. Surf. Sci. 2015, 324, 854-863. [CrossRef] 
30. Karthika, J.S.; Vishalakshi, B. Novel stimuli responsive gellan gum-graft-poly (DMAEMA) hydrogel as adsorbent for anionic dye. Int. J. Bio. Macromol. 2015, 81, 648-655. [CrossRef]

31. Ramesha, G.K.; Kumara, A.V.; Muralidhara, H.B.; Sampath, S. Graphene and graphene oxide as effective adsorbents toward anionic and cationic dyes. J. Colloid Interface Sci. 2011, 361, 270-277. [CrossRef] [PubMed]

32. Tan, J.; Song, Y.; Huang, X.; Zhou, L. Facile functionalization of natural peach gum polysaccharide with multiple amine groups for highly efficient removal of toxic hexavalent chromium (Cr (VI)) ions from water. ACS Omega 2018, 3, 17309-17318. [CrossRef] [PubMed]

33. Deng, J.H.; Zhang, X.R.; Zeng, G.M.; Gong, J.L.; Niu, Q.Y.; Liang, J. Simultaneous removal of Cd(II) and ionic dyes from aqueous solution using magnetic graphene oxide nanocomposite as an adsorbent. Chem. Eng. J. 2013, 226, 189-200. [CrossRef]

34. Gong, J.L.; Wang, B.; Zeng, G.M.; Yang, C.P.; Niu, C.G.; Niu, Q.Y.; Zhou, W.J.; Liang, Y. Removal of cationic dyes from aqueous solution using magnetic multi-wall carbon nanotube nanocomposite as adsorbent. J. Hazard. Mater. 2009, 164, $1517-1522$. [CrossRef]

35. Wu, D.; Zheng, P.; Chang, P.R.; Ma, X. Preparation and characterization of magnetic rectorite/iron oxide nanocomposites and its application for the removal of the dyes. Chem. Eng. J. 2011, 174, 489-494. [CrossRef]

36. Abdulla, N.K.; Siddiqui, S.I.; Tara, N.; Hashmi, A.A.; Chaudhry, S.A. Psidium guajava leave-based magnetic nanocomposite $\gamma-\mathrm{Fe}_{2} \mathrm{O}_{3} @ \mathrm{GL}$ : A green technology for methylene blue removal from water. J. Environ. Chem. Eng. 2019, 7, 103423. [CrossRef]

37. Xie, G.; Xi, P.; Liu, H.; Chen, F.; Huang, L.; Shi, Y.; Hou, F.; Zeng, Z.; Shao, C.; Wang, J. A facile chemical method to produce superparamagnetic graphene oxide- $\mathrm{Fe}_{3} \mathrm{O}_{4}$ hybrid composite and its application in the removal of dyes from aqueous solution. $J$. Mater. Chem. 2012, 22, 1033-1039. [CrossRef]

38. Ma, Y.Z.; Zheng, D.F.; Mo, Z.Y.; Dong, R.J.; Qiu, X.Q. Magnetic lignin-based carbon nanoparticles and the adsorption for removal of methyl orange. Colloid. Surf. A 2018, 559, 226-234. [CrossRef]

39. Mallakpour, S. Hatami, M. An effective, low-cost and recyclable bio-adsorbent having amino acid intercalated $\mathrm{LDH}_{\mathrm{F}} \mathrm{Fe}_{3} \mathrm{O}_{4} / \mathrm{PVA}$ magnetic nanocomposites for removal of methyl orange from aqueous solution. Appl. Clay Sci. 2019, 174, 127-137. [CrossRef]

40. Liu, D.M.; Dong, C.; Zhong, J.; Ren, S.; Chen, Y.; Qiu, T. Facile preparation of chitosan modified magnetic kaolin by one-pot coprecipitation method for efficient removal of methyl orange. Carbohydr. Polym. 2020, 245, 116572. [CrossRef]

41. Duan, Y.; Song, Y.; Zhou, L. Facile synthesis of polyamidoamine dendrimer gel with multiple amine groups as a super adsorbent for highly efficient and selective removal of anionic dyes. J. Colloid Interface Sci. 2019, 546, 351-360. [CrossRef] [PubMed]

42. Alver, E.; Metin, A.Ü.; Brouers, F. Methylene blue adsorption on magnetic alginate/rice husk bio-composite. Int. J. Bio. Macromol. 2020, 154, 104-113. [CrossRef] [PubMed] 